\title{
A molecular assay to predict survival in resected non-squamous non-small-cell lung cancer
}

This 14-gene expression assay that uses quantitative PCR on formalin-fixed paraffin-embedded tissue samples was developed and validated and compared with conventional staging. This assay was developed in patients with resected non-squamous non-small-cell lung cancer (NSCLC) at one centre, and then validated in patients with resected stage I non-squamous NSCLC and on patients with resected stage I-III nonsquamous NSCLC in two further centres.

This assay was the strongest predictor of 5-year mortality compared with clinical risk factors such as sex, age, smoking status, tumour size and even disease stage. It also outperformed criteria used to identify high-risk patients with stage I disease and differentiated low-risk, intermediate-risk and high-risk patients within all disease stages. However, this assay did not take account of the relationship between genes, nor redundancy of information, and the combination of top-ranked features might not be optimal. Additionally, lung cancers with different amounts of cancer stem cells should be considered.

Although most studies focus on the prediction of signatures, the biological relevance of genes in the signature is important. More detailed clinical risk factors, such as high-tech imaging studies or cardiopulmonary exercise testing might be an option for prognosis and prediction. Prognostic and predictive markers will allow personalised medicine for patients. Further studies will tell whether this assay is of functional relevance.

- Kratz JR, He J, Van Den Eeden SK, et al. A practical molecular assay to predict survival in resected non-squamous, non-small-cell lung cancer: development and international validation studies. Lancet 2012;379:823-32.

Tin Myo Hlaing, Jeng-Shing Wang

Correspondence to Dr Jeng-Shing Wang, Antai Medical Care Cooperation Antai Tian-Sheng Memorial Hospital, Department of Thoracic Medicine, Pingtung, \& Department of Senior Citizen Service Management, Yuh-Ing Junior College, Kaohsiung, Pingtung 928, Taiwan; wangjs6@hotmail.com 


\section{Correction}

Hlaing TM, Wang J-S. A molecular assay to predict survival in resected non-squamous non-small-cell lung cancer. Thorax 2013;68:47 doi:10.1136/thoraxjnl-2012-202055.

The correspondence address for Jeng-Shing Wang should read: Dr Jeng-Shing Wang, Antai Medical Care Cooperation Antai Tian-Sheng Memorial Hospital, Department of Thoracic Medicine, Pingtung, and Department of Internal Medicine, Taipei Medical University, Taipei, Pingtung 928, Taiwan; wangjs6@hotmail.com.

Thorax 2013;68:596. doi:10.1136/thoraxjnl-2012-202055corr1 